\title{
ATRESIA BRONQUIAL CONGÉNITA: UNA SERIE DE CASOS EN POBLACIÓN ADULTA COLOMBIANA
}

Diego Fernando García-Bohórquez, Andrés Villabona-Rueda, Javier Enrique Fajardo-Rivero. Grupo de investigación MEDITA, Universidad Industrial de Santander.

\section{INTRODUCCIÓN}

La atresia bronquial $(\mathrm{AB})$ es una anomalía congénita rara de etiología poco clara. Se clasifica en proximal o periférica. La afectación proximal es mortal, en la vida intrauterina o en el recién nacido, mientras que la periférica, la mayoría de las veces es asintomática y el hallazgo tiende a ser incidental en las ayudas diagnósticas. Al ser una patología de poca incidencia a nivel mundial y en nuestro medio, presentamos una serie de casos con el objetivo de comparar la clínica y localización con lo reportado en la literatura.

\section{CASOS CLÍNICOS}

Caso 1: Hombre de 34 años que tras encontrar disminución del tamaño pulmonar izquierdo sin alteraciones parenquimatosas en una radiografía de tórax (RT) de control es remitido al servicio de neumología. En la TC se encontraron hallazgos compatibles con un broncocele, e hiper-aireación del segmento apicoposterior del lóbulo superior izquierdo, lo que sugirió una $\mathrm{AB}$ allí.

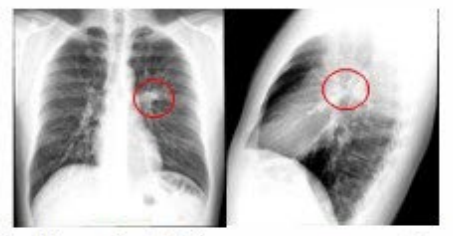

Imagen 1. Radiografía de tórax antero-posterior y lateral. EI círculo rojo muestra una atelectasia parahiliar. izquierda.

Caso 2: Hombre de 33 años. Consulta por tos intensa y paroxística. En la RT se observó una lesión focal "en dedo de guante" en la región parahiliar derecha. La TC presentó lesión ovalada en segmento superior de lóbulo inferior derecho con densidad cálcica en su interior, de aspecto benigno y atenuación en mosaico generalizada. Los hallazgos observados sugirieron una $A B$ en el segmento superior del lóbulo inferior derecho.

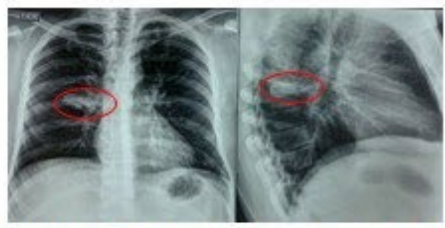

Imagen 2. Radiografía de tórax antero-posterior y lateral. El círculo rojo muestra una lesión focal "en dedo de guante" parahiliar derecha..
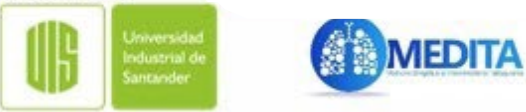

Caso 3: Hombre 26 años. Consulta por dolor intermitente en hemitórax izquierdo, de 3 meses de evolución. En la TC se observó imagen nodular irregular parahiliar derecha con hiperinflación segmentaria distal a la alteración.
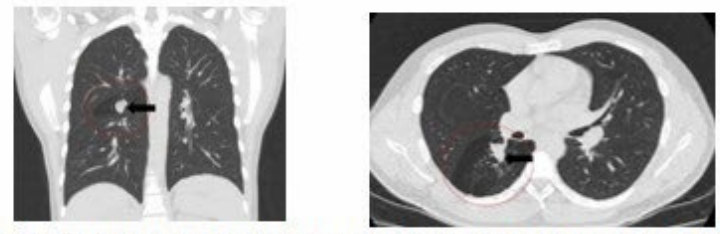

Imagen 3. TAC: El círculo enmarca una región hipodensa en el lóbulo superior derecho. La flecha evidencia el mucocele

\section{DISCUSIÓN/ CONCLUSIÓN}

La AB es poco frecuente. Todos los casos reportados fueron pacientes masculinos concorde a lo reporta de relación hombre - mujer es 2:1. El diagnostico usualmente es incidental ya que aproximadamente el $50 \%$ de los pacientes son asintomáticos, en la serie presentada, un caso tuvo dolor torácico persistente en el hemitórax derecho, sin embargo no pudo establecerse la relación directa con dicha patología. Se ha reportado que el bronquio más frecuentemente afectado es el apical posterior del lóbulo superior izquierdo, como en el primer caso reportado. Le sigue el lóbulo inferior izquierdo y el lóbulo medio e inferior derecho, ubicación del segundo caso aquí reportado. El tercero de nuestros casos tiene una disposición atípica afectando el lóbulo superior derecho. Aunque la $\mathrm{AB}$ en su mayoría es descrita como hallazgo aislado también puede encontrarse asociada a otras malformaciones. En nuestros casos no hubo hallazgos o reportes de malformaciones adicionales.

La serie de casos presentada permitió realizar una comparación con los aspectos de la $\mathrm{AB}$ descritos en la literatura, lo cual no estuvo lejos del estándar, y nos proporciona información relevante para considerar esta patología en el diagnóstico diferencial de la afectación bronquial focal con hiperinsuflación pulmonar distal. 1. Higuchi R, Goto T, Nakagomi T, Oyama T. Surgery for congenital bronchial atresia. Asian Cardiovasc Thorac Ann 2018;26(6):485-8.

2. Acosta Gordillo L, Marquez Fernandez J, Medina Gil MC, Carrasco Azcona MA, Andres Martin A. [Asymptomatic congenital bronchial atresia in a 10-year-old boy]. An Pediatr Barc 2005;62(4):386-8.

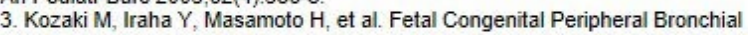
Atresia Diagnosed by Magnetic Resonance Imaging: Two Case Reports. A.JP Rep 2018;8(4):e201-e5. 